\title{
Construção de um luxímetro digital utilizando plataforma Arduino para uso em laboratórios didáticos
}

Arduino based digital luxmeter for didactical laboratory use

\author{
C. B. Pereira ${ }^{1}$, S. L. M. Berleze ${ }^{1}$, W. A. Soares ${ }^{1}$, J. P. M. Serbena ${ }^{*} @$ \\ ${ }^{1}$ Universidade Federal do Paraná, Departamento de Física, Centro Politécnico, Curitiba, PR, Brasil.
}

\begin{abstract}
Recebido em 04 de dezembro de 2020. Revisado em 16 de janeiro de 2021. Aceito em 25 de janeiro de 2021.
Este trabalho teve como finalidade a construção de um luxímetro utilizando um fotodetector/conversor luz em frequência (LTF, do inglês "light to frequency") e plataforma Arduino. As etapas de execução consistiram no desenvolvimento do código de programação da comunicação e controle entre fotodetector e Arduino, na confecção de uma placa de circuito impresso caseira e no acabamento do luxímetro com a inserção de botões, display e demais componentes eletrônicos numa caixa Patola®. O luxímetro construído foi utilizado em três experimentos didáticos realizados na disciplina de laboratório dos cursos de graduação em Física e Engenharias cujo tema estudado é a Óptica. As medidas realizadas demonstraram que o luxímetro atende à necessidade do laboratório ao substituir equipamentos comerciais atualmente utilizados.
\end{abstract}

Palavras-chave: Óptica, difração em fenda única, luxímetro, Arduino.

This work aimed the construction of a luxmeter using a photodetector/light to frequency converter (LTF) and Arduino platform. The execution steps consisted on developing the communication and control programming code between photodetector and Arduino, manufacturing a homemade printed circuit board and finishing the assembled luxmeter with the insertion of buttons, display and all other electronic components in a Patola ${ }^{\circledR}$ box. The built luxmeter was used in three didactic experiments carried out in the undergraduate laboratory discipline of Physics and Engineering courses whose studied topic is Optics. The performed measurements demonstrated that the luxmeter meets the laboratory's needs when replacing commercial equipments that are currently in use. Keywords: Optics, single slit diffraction, luxmeter, Arduino.

\section{Introdução}

Nos livros didáticos de Física Básica de graduação em Ensino Superior que abordam o tema Óptica, a difração é apresentada como um fenômeno associado à chamada ótica física, cujos efeitos óticos são explicados utilizando-se das propriedades da natureza ondulatória da luz [1]. Esse fenômeno é normalmente abordado tanto teoricamente quanto experimentalmente, visto que, além de sua importância para a compreensão de conceitos físicos mais avançados, é um fenômeno que pode ser facilmente observado e replicado na prática. Isto se deve à atual disponibilidade de fontes de luz laser baratas e acessíveis, sendo utilizados os mais diversos elementos do cotidiano como aberturas ou obstáculos para a difração da luz, desde CDs e tecidos a fios de cabelo. Uma boa revisão dos diferentes materiais e formas de se observar a difração é reportada por Ribeiro et al. em [2]. Vale ressaltar que neste trabalho, quando houver referência à difração da luz, será considerada a difração de Fraunhofer ou de campo longínquo, na qual as ondas incidente e difratada são aproximadamente planas sobre a região das aberturas ou obstáculos difratantes. Nesta

\footnotetext{
*Endereço de correspondência: serbena@fisica.ufpr.br
}

situação já não é mais possível reconhecer a abertura ou obstáculo, o padrão de difração cobre uma região extensa e qualquer variação entre distâncias tem como única consequência a variação do tamanho do padrão mas não da sua forma [3].

Uma atividade muito interessante, do ponto de vista experimental, é a obtenção da curva de intensidade luminosa em função do ângulo entre o eixo óptico e a linha que conecta a abertura ou obstáculo a um determinado ponto de observação do padrão de difração formado. Para as medidas experimentais, deve-se utilizar um sonda de medição relativamente pequena, da ordem de centenas de micrometros de diâmetro, para não impactar na resolução da medida da distância, o que pode ser conseguido com uma fibra óptica. Contudo, a intensidade luminosa do máximo secundário não chega a $5 \%$ da intensidade luminosa do máximo central, o que faz com que seja necessária a utilização ou de luxímetros de alta sensibilidade ou de fontes luminosas de alta potência para a coleta das medidas experimentais. A segundo opção proporciona um risco maior de acidentes, algo a ser seriamente considerado num laboratório didático, sendo a primeira opção normalmente escolhida. Isso implica num aumento de custos do experimento, visto que os luxímetros são mais caros quanto maior sua resolução. 


\section{Princípios do Projeto}

Particularmente no laboratório didático de Física Experimental IV do Departamento de Física da Universidade Federal do Paraná, a experiência "Difração da Luz" é realizada utilizando-se um luxímetro analógico PASCO ${ }^{\circledR}$ OS-8020 de alta sensibilidade (resolução máxima aproximadamente 0,002 lux) 4. Especificamente este luxímetro é um modelo obsoleto, não sendo mais comercializado, o que impede uma possível substituição pelo mesmo modelo e encarece sua manutenção por necessitar de mão-de-obra especializada. Adicionalmente, estão operando no limite de sua vida útil. Luxímetros digitais com esta mesma resolução podem ser encontrados, mas a preços muitas vezes não acessíveis, como o modelo 07137-00 da PHYWE, cujo custo gira em torno de 650 Euros. Luxímetros mais baratos usualmente não possuem resolução suficiente para a realização deste experimento específico. Assim, a proposta deste trabalho é a substituição do luxímetro PASCO® OS-8020 por uma alternativa mais barata e que possa permitir a manutenção no próprio laboratório, possibilitando o treinamento de alunos da graduação em eletrônica e programação.

Dentre as inúmeras possibilidades, optou-se pela utilização da plataforma Arduino pois é uma plataforma abundantemente difundida, simples, de baixo custo e de código livre, o que proporciona facilidade de uso, de melhoramentos e de reparos, principalmente devido ao fato de se trabalhar de modo modular. Sua linguagem de programação, baseada em $\mathrm{C} / \mathrm{C}++$, é normalmente ensinada nas aulas de programação de computadores dos cursos de graduação, facilitando ainda a familiarização dos discentes com a instrumentação. Uma excelente revisão sobre as contribuiçoes da plataforma Arduino no ensino de Física foi reportada por Moreira et al. em [5].

Contudo, o elemento central para o equipamento funcionar como desejado é o dispositivo fotosensível escolhido. Pedroso et al. construíram um luxímetro digital utilizando um transistor NPN de alta potência 2N3055 desencapado como elemento fotosensível [6]. Apesar do baixo custo e facilidade de construção, dificilmente tal dispositivo teria resolução suficiente para ser aplicado com o propósito deste trabalho. Por isso, optou-se pela abordagem publicada por Patrik [7, que faz uso de um fotodetector/conversor luz em frequência (LTF, do inglês "light to frequency") TSL237s-LF [8]. Resumidamente, um circuito desse tipo usa uma versão CMOS do temporizador 555 para formar um conversor de intensidade luminosa em frequência e um pequeno fotodiodo como detector de luz. A corrente de fuga do fotodiodo reversamente polarizado é proporcional à intensidade da luz incidente. Esta corrente de fuga carrega um capacitor de $0,01 \mu \mathrm{F}$. Quando a tensão do capacitor atinge $2 / 3$ da tensão de alimentação, a tensão de saída do circuito diminui. Como resultado, o capacitor descarrega através do fotodiodo. Quando a tensão do capacitor atinge 1/3 da tensão de alimentação, a tensão de saída aumenta, iniciando outra carga no capacitor. Esse ciclo continua, obtendo-se uma frequência na saída do circuito que é proporcional à intensidade da luz incidente no fotodiodo. Normalmente, a frequência do oscilador varia desde aproximadamente $1 \mathrm{~Hz}$ na escuridão total até cerca de $25 \mathrm{kHz}$ na luz solar. Outros extremos de frequência são possíveis alterando-se o valor de capacitância do capacitor. Detalhes desse tipo de fotodetector podem ser encontrados, por exemplo, em [9, 10. O fato é que a saída digital permite uma interface direta com um microcontrolador ou outro circuito lógico, no caso, o Arduino.

Com isso em mente, pode-se construir um luxímetro. Na literatura [1] são apresentadas equações para a intensidade da luz emitida por uma fonte ou um sistema óptico, a qual pode depender da direção de propagação. Por sua vez, os luxímetros medem uma grandeza chamada iluminância. A intensidade luminosa e a iluminância fazem parte do sistema fotométrico, que se aplica no espectro visível e considera a sensação visual de um observador humano. É importante, então, conhecer como são definidas estas grandezas e de que forma elas estão relacionadas.

Uma fonte ou sistema óptico pode emitir certa quantidade de energia ao longo do tempo. A taxa de transferência dessa energia é chamada de fluxo luminoso, que é medido em lúmens ( $\mathrm{lm}$ ) no SI. O fluxo luminoso pode ser diferente para cada direção de propagação da luz a partir da fonte. O fenômeno da difração da luz é um exemplo, onde tem-se a variação entre máximos e mínimos. Em seguida, é necessário considerar uma região delimitada por uma superfície cônica imaginária com vértice no centro da fonte e eixo ao longo de uma dada direção. Esta superfície cônica subtende no seu interior um ângulo sólido. A intensidade luminosa da fonte numa certa direção é definida como o fluxo luminoso total naquela direção por unidade de ângulo sólido. Sua unidade no SI é o candela (cd). Outra grandeza importante é definida a partir do fluxo luminoso que incide sobre uma unidade de área. Como a superfície do sensor é receptora de luz, esta densidade superficial de fluxo define uma grandeza chamada iluminância, cuja unidade no SI é o lux (lx).

A equação que relaciona a intensidade luminosa e a iluminância contém integrais de superfície, cuja resolução pode ser difícil dependendo da geometria do sistema. Entretanto, quando o sensor está afastado da fonte e tem uma área pequena, os raios incidentes sobre ele são praticamente paralelos e a solução é bastante simples. Estas condições são satisfeitas nos experimentos realizados no laboratório de ensino. Considerando ainda que os raios incidam perpendicularmente à superfície do sensor, a iluminância dependerá da intensidade emitida pela fonte e da distância entre ambos seguindo a lei fotométrica da distância. Se a distância entre o sensor e a fonte for mantida constante, a iluminância dependerá apenas da intensidade luminosa, numa proporção direta. 
Esta relação permite o estudo de diferentes fenômenos a partir das medidas com o luxímetro.

\section{Construção do Luxímetro}

A parte central do equipamento proposto reside no fotodetector TSL237s-LF. Para que ele possa operar corretamente, o fabricante recomenda que as linhas de alimentação do sensor sejam desacopladas do fotodetector por um capacitor cuja capacitância esteja entre 0,01 $\mu \mathrm{F}$ e $0,1 \mu \mathrm{F}$, com os terminais do capacitor conectados o mais próximo possível do TSL237s-LF [8]. Assim, o luxímetro construído consiste nos seguintes componentes: Arduino Nano, fotodetector TSL237s-LF, capacitor de $0,1 \mu \mathrm{F}$, botão liga-desliga, dois botões para medida (um para armazenamento da leitura residual e outro para leitura) e display LCD $16 \times 2$ para leitura da medida pelo experimentador, além de dois LEDs indicadores de "leitura em processo" e "pronto para leitura". Para as aplicações desejadas neste trabalho, não há necessidade de obter valores calibrados da iluminância incidente, uma vez que estas serão normalizadas, conforme explicado na próxima sessão. Ainda assim, foi necessário inserir um botão adicional para medida da iluminância residual. Essa medida é armazenada na memória interna do Arduino e todas as leituras subsequentes têm este valor subtraído. Isso permite eliminar contribuições da luz ambiente, permitindo que os experimentos sejam realizados na penumbra.

Toda a montagem foi feita, inicialmente, utilizandose um protoboard, como apresentado na Figura 1 1 , cujo esquema do circuito eletrônico é apresentado na Figura 1 b. Nesta etapa, foi implementado o código de comunicação entre os diferentes componentes, em especial entre o TSL237s-LF e o Arduino. Para que possa ser apresentado um valor razoável (mas não calibrado) de iluminância, foi necessário inserir no código a seguinte linha de comando [].

$$
E=\frac{f_{0}-f_{D}}{R}
$$

onde $E$ é iluminância, $f_{0}$ e $f_{D}$ são as frequências de leitura e residual, respectivamente, e $R$ é uma constante relacionada à responsividade do TSL237s-LF [8]. A menos que o luxímetro seja calibrado, o valor de $R$ é arbitrário. Por simplicidade, foi utilizado o valor $2,3 \mathrm{kHz} / \mu \mathrm{lux}$, sugerido por Patrik [7]. A rigor, numa equação similar à 1 relacionam-se as grandezas irradiância, frequência e responsividade ${ }^{1}$ Contudo, uma vez que serão utilizados

\footnotetext{
${ }^{1}$ Irradiância é uma grandeza radiométrica, enquanto iluminância é sua equivalente fotométrica. As grandezas fotométricas são obtidas levando-se em conta uma função de eficiência luminosa espectral fotópica $V(\lambda)$, definida pela Commission International de l'Éclairage (CIE), com o intuito de quantificar o efeito visual produzido pela radiação ótica como é percebida pelo olho humano. $\mathrm{Ou}$ seja, essencialmente considera somente a região visível do espectro eletromagnético.
}

a)

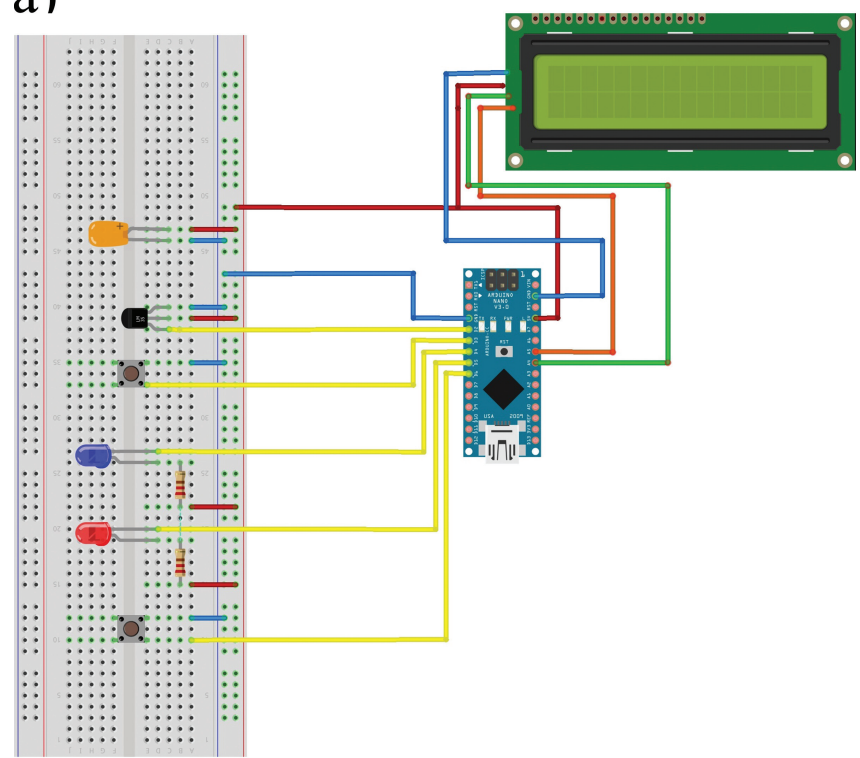

b)

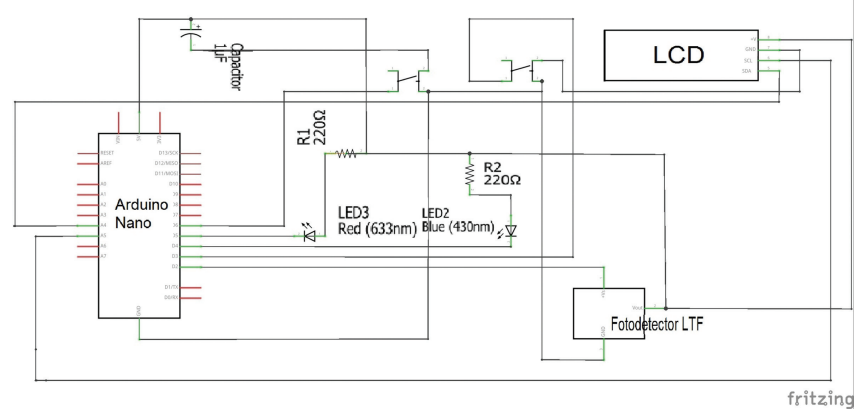

Figura 1: a) Montagem do luxímetro utilizando o protoboard; b) esquema do circuito eletrônico construído.

valores relativos nos experimentos, que a responsividade espectral do fotodetector TSL237s-LF 8 não difere muito daquela do fotodetector no luxímetro PASCO ${ }^{\circledR}$ OS-8020 4] e que os valores absolutos medidos pelos luxímetros comerciais só têm validade nos experimentos quando é utilizada a mesma fonte de sua calibração (normalmente esta é citada no manual e está presente nos kits comercializados), pode-se considerar a validade da equação 1 da maneira acima colocada.

$\mathrm{Na}$ sequência foi projetada uma placa de circuito impresso, em substituição ao protoboard, utilizando-se o software gratuito PCB Artist, conforme a Figura 2 . Para sua confecção foi utilizada uma placa virgem de circuito impresso, de fenolite e com face simples, dimensões $10,2 \mathrm{~cm} \times 9,0 \mathrm{~cm}$. Inicialmente, a superfície de cobre da placa foi limpa com uma esponja de lã de aço fina. O desenho do circuito, com suas trilhas e ilhas foi impresso em papel glossy numa impressora a laser comum. Em seguida, este desenho foi transferido para a placa usando o método térmico. Para isso colocou-se a face impressa do papel em contato com o cobre da placa e cobriu-se com um pedaço de papel sulfite. Passou-se com 

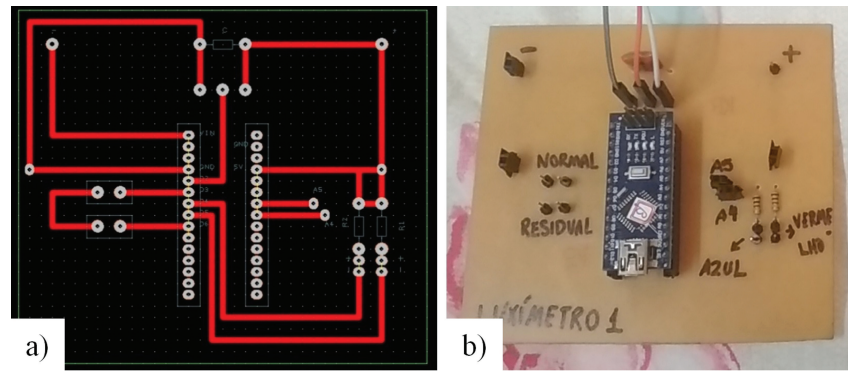

Figura 2: a) Projeto da placa de circuito impresso feita no programa PCB Artist. b) Foto da placa de circuito impresso finalizada, com os componentes soldados a ela.

o ferro de passar roupa aquecido, fazendo leve pressão, de modo que o tonner do desenho ficasse aderido à placa. Esperou-se esfriar completamente, então o papel foi umedecido e depois retirado lentamente, deixando o tonner que compõe o desenho aderido à placa.

Feito isso, mergulhou-se a placa de fenolite com o circuito transferido numa solução de percloreto de ferro para ocorrer a corrosão das partes não cobertas do cobre. Terminada a corrosão, lavou-se a placa em água corrente. Mantendo-a molhada e usando uma esponja macia e sabão, o tonner foi removido deixando visível as trilhas de cobre. Após a placa estar seca, foram feitos os furos com uma mini-furadeira de mão. As trilhas de cobre foram limpas com uma esponja de aço fina até o metal ficar completamente exposto e, em seguida, foi aplicada uma demão de verniz caseiro preparado com breu e álcool isopropílico. Para finalizar foram soldados os componentes e pinos conectores conforme a Figura $2 \mathrm{~b}$.

Furos também foram feitos na caixa Patola ${ }^{\circledR}$ usada para abrigar os componentes eletrônicos, botões, LEDs, display LCD e sensor TSL237s-LF. Optou-se por acoplar a entrada de luz do TSL237s-LF a uma conexão de fibra ótica usada na comunicação de áudio em DVDs. Isso permite uma certa mobilidade e flexibilidade na sonda de medição sem perda significativa de sensibilidade. Dessa forma, o TSL237s-LF foi adaptado a um conector $\mathrm{BNC}$ fêmea de painel e fixado à caixa Patola ${ }^{\circledR}$ com cola instantânea Vonder e bicarbonato de sódio, como mostrado nas Figuras 3a e 3b. Para esta montagem foi necessário extrair a conexão elétrica e a parte isolante central do conector original. Uma das extremidades da fibra ótica foi fixada a um conector BNC macho, do qual também foram retirados o pino e o isolante central, como ilustrado na Figura 34. Com isso a fibra ótica pode ser facilmente conectada ao sensor TSL237s-LF. A sua outra extremidade, livre de conexões, se torna a sonda de medição. A Figura 3 d mostra a caixa Patola® fechada com todo o circuito dentro e o luxímetro finalizado pode ser observado na Figura 3 .

\section{Aplicação em Alguns Experimentos}

Como dito anteriormente, a motivação para a construção deste luxímetro é o levantamento experimental
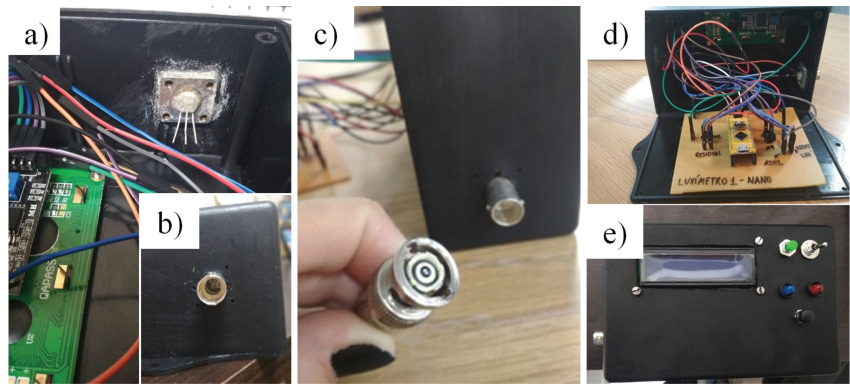

Figura 3: a) Fixação do TSL237s-LF junto a um conector BNC fêmea na caixa Patola $\AA$; b) visão exterior da conexão do TSL237s-LF; c) fibra óptica com o conector BNC macho para fácil acoplamento da sonda de medição; d) circuito e conexões internas do luxímetro finalizado; e) exterior do luxímetro finalizado.

da intensidade da luz monocromática difratada por uma fenda única em função da posição com relação ao máximo central da difração. Usualmente o experimento da difração da luz visível em fenda única é conteúdo das disciplinas de laboratório dos cursos de graduação em Física e das Engenharias. Para facilitar a visualização e as medidas, é frequente o uso de lasers que produzem luz monocromática com intensidades razoáveis. Contudo, mesmo assim, é necessário um luxímetro suficientemente sensível para possibilitar a medida da intensidade luminosa, especialmente dos máximos secundários, cujas intensidades não chegam a $5 \%$ da intensidade do máximo central.

No caso mais simples, da difração da luz monocromática por um fenda única de geometria retangular, na qual uma das dimensões da abertura é muito maior que a outra, esta curva de intensidade é normalmente deduzida e apresentada nos livros didáticos de física básica, tendo uma forma matematicamente simples cuja expressão é dada por:

$$
\frac{I}{I_{0}}=\left(\frac{\sin (\alpha)}{\alpha}\right)^{2}
$$

onde $I$ é a intensidade luminosa, $I_{0}$ é a intensidade luminosa do máximo central e $\alpha$ é dado por:

$$
\alpha=\frac{\pi \cdot a \cdot \sin (\theta)}{\lambda}
$$

onde $a$ é a largura da fenda, $\lambda$ é o comprimento de onda da luz difratada e $\theta$ é o ângulo formado entre o eixo óptico e a linha que conecta a abertura ou obstáculo ao ponto de observação. A verificação experimental desta expressão matemática pode ser facilmente realizada ao concentrar a análise em pontos próximos ao máximo central da figura de difração. Considerando ainda uma distância entre fenda e fibra ótica $L$ muito maior que a distância entre o máximo central da figura de difração e a posição de observação $y, L>>y$, pode-se fazer a aproximação $\sin (\theta) \approx \tan (\theta) \approx y / L$, o que leva à seguinte expressão para $\alpha$, agora dependente diretamente 
de $y$ :

$$
\alpha=\frac{\pi \cdot a}{L \cdot \lambda} \cdot y
$$

o que faz com que a equação 2 tome a seguinte forma:

$$
\frac{I}{I_{0}}=\left(\frac{\sin ((\pi \cdot a) /(L \cdot \lambda) \cdot y)}{(\pi \cdot a) /(L \cdot \lambda) \cdot y}\right)^{2}
$$

Assim, tem-se uma expressão que relaciona diretamente a intensidade luminosa em função da posição para a figura de difração obtida.

Como forma de verificar se o luxímetro construído pode substituir o PASCO® OS-8020 nas aulas, foi montado o experimento "Difração da Luz em Fenda Única". Neste experimento, apresentado na Figura 4 a e realizado numa sala escura, luz com comprimento de onda $633 \mathrm{~nm}$ proveniente de um laser de He-Ne JDSU modelo 1508-1 e potência $<1 \mathrm{~mW} 12$ incide numa fenda de largura nominal igual a 0,04 mm PASCO® OS8850 [13, cuja figura de difração é projetada sobre um suporte da sonda de fibra óptica a $0,5 \mathrm{~m}$ de distância. Todo o experimento é montado num trilho óptico. Inicialmente, acopla-se a fibra óptica ao luxímetro e a um transladador horizontal em sua posição central. O transladador é capaz de medir a posição da fibra óptica num intervalo de aproximadamente $50 \mathrm{~mm}$, com precisão de $1 \mathrm{~mm}$. Dessa maneira, é possível medir a intensidade luminosa em função da posição da fibra ótica. Em seguida, a fonte laser é posicionada no trilho óptico a uma distância de $70 \mathrm{~cm}$ da fibra óptica. O laser é ligado e ajustado para incidir diretamente sobre a fibra óptica. Com isso, o máximo central da difração coincide com esta posição, medida no transladador. Assim, é possível obter tanto uma curva simétrica com relação ao máximo central quanto a iluminância dos dois primeiros máximos secundários. Entre o laser e a fibra óptica é inserida a fenda única, a uma distância de $50 \mathrm{~cm}$ desta última, ajustando-a de tal forma a se obter uma figura de difração orientada horizontalmente. Isto permite que a fibra óptica possa percorrê-la. Na sequência, com o laser desligado, é feita a medida residual para descontar contribuições da luz ambiente. Liga-se novamente o laser e, a partir deste momento, medidas da iluminância da luz difratada são feitas transladando-se a fibra óptica de $2 \mathrm{~mm}$ em $2 \mathrm{~mm}$, até que percorra todo seu trajeto disponível conforme a Figura 4 b.

Uma vez coletados todos os dados, dividem-se os valores da iluminância pelo valor máximo medido, obtendose assim uma medida relativa em função da posição. Os resultados obtidos são apresentados na Figura 5 . onde o valor de posição zero foi arbitrariamente escolhido como o ponto de máxima iluminância.

A linha tracejada na Figura 5 é o ajuste da curva teórica representada pela equação 5 , de onde é possível extrair o valor da largura da fenda. Para realizar este ajuste, foi considerada a seguinte equação no processo
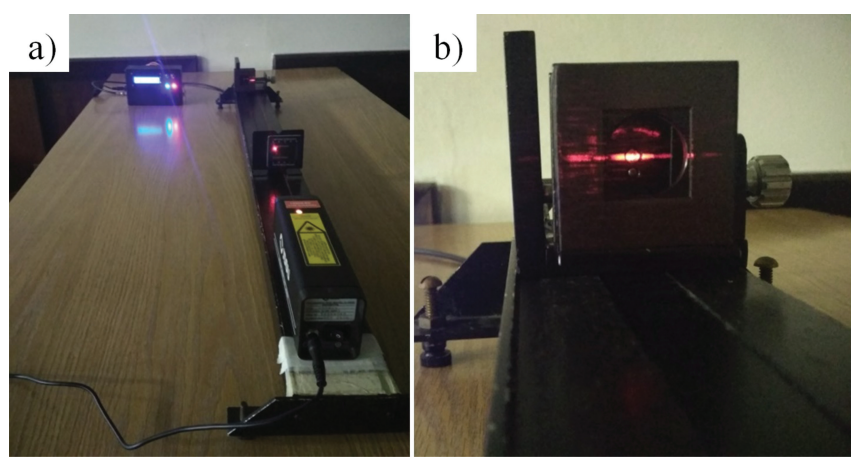

Figura 4: a) Foto da montagem experimental utilizada; b) detalhe da figura de difração incidindo sobre o suporte com a fibra óptica, a qual pode se movimentar horizontalmente.

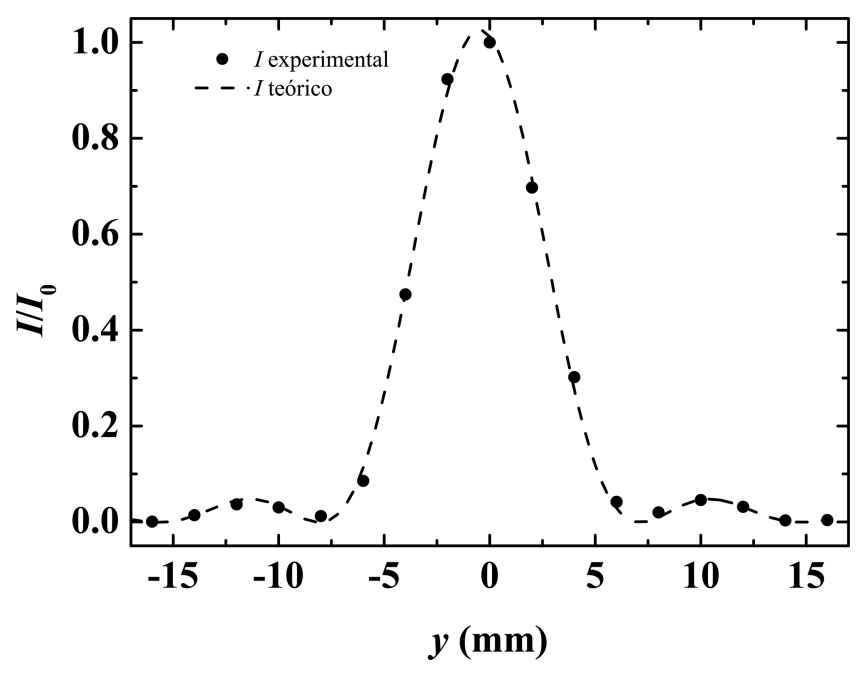

Figura 5: Curva de intensidade relativa em função da posição. Os pontos sólidos circulares são as medidas experimentais e a linha tracejada é o ajuste teórico.

de regressão por mínimos quadrados:

$$
I / I_{0}=\mathrm{A}+\mathrm{B} \cdot\left[\frac{\sin (\mathrm{C} \cdot(y+\mathrm{D}))}{\mathrm{C} \cdot(y+\mathrm{D})}\right]^{2}
$$

onde A, B, C e D são constantes a determinar.

A constante A ajusta o valor mínimo de intensidade de luz no ambiente e é esperado que seja o mais próximo de zero. O botão para leitura de intensidade residual permite a obtenção de valores bem baixos para A. A constante B ajusta o valor máximo da intensidade que, neste caso de intensidade relativa, é esperado que seja próximo a um. O valor da constante $\mathrm{D}$ ajusta a posição em que ocorre o máximo de intensidade relativa, esperado próximo a zero. Como a intensidade é normalizada utilizando os pontos experimentais, é possível que B tenha uma leve diferença da unidade e D uma leve diferença do zero, implicando que o valor máximo da intensidade relativa ajustada não se encontra na posição zero e, consequentemente, é maior que a unidade. Finalmente, a constante $\mathrm{C}$ depende do sistema 
e é a partir de seu valor que se pode calcular a largura da fenda, uma vez que ela representa a seguinte expressão:

$$
\mathrm{C}=\frac{\pi \cdot a}{L \cdot \lambda}
$$

Os valores obtidos são:

$$
\begin{aligned}
& A=(-1 \pm 4) \times 10^{-3} \\
& B=1,03 \pm 0,01 \\
& C=(413 \pm 4) \mathrm{m}^{-1} \\
& D=(47 \pm 3) \times 10^{-4} \mathrm{~m}
\end{aligned}
$$

A largura da fenda obtida dessa forma é igual a 0,04158 mm, uma variação percentual de apenas 3,9\% se comparado com o valor nominal.

Para verificar sua viabilidade nas demais atividades do laboratório didático, o luxímetro construído foi utilizado também nos experimentos que tratam sobre a polarização da luz e a lei fotométrica da distância. No primeiro, é verificada experimentalmente a Lei de Malus, que descreve como a intensidade $I$ da luz transmitida através de dois polarizadores depende do ângulo $\theta$ entre seus eixos de polarização. Matematicamente, a Lei de Malus é expressa como:

$$
I=I_{0} \cdot \cos ^{2}(\theta)
$$

onde $I_{0}$ será considerada como a intensidade máxima da luz emergente no segundo polarizador. A figura 6 mostra o gráfico obtido a partir da coleta de dados realizada utilizando-se o luxímetro construído, utilizando-se como fonte de luz uma lâmpada LED branca.

O ajuste aos dados experimentais foi feito através da seguinte equação:

$$
I / I_{0}=\mathrm{A}^{\prime}+\mathrm{B}^{\prime} \cdot \cos ^{2}\left(\theta+\mathrm{C}^{\prime}\right)
$$

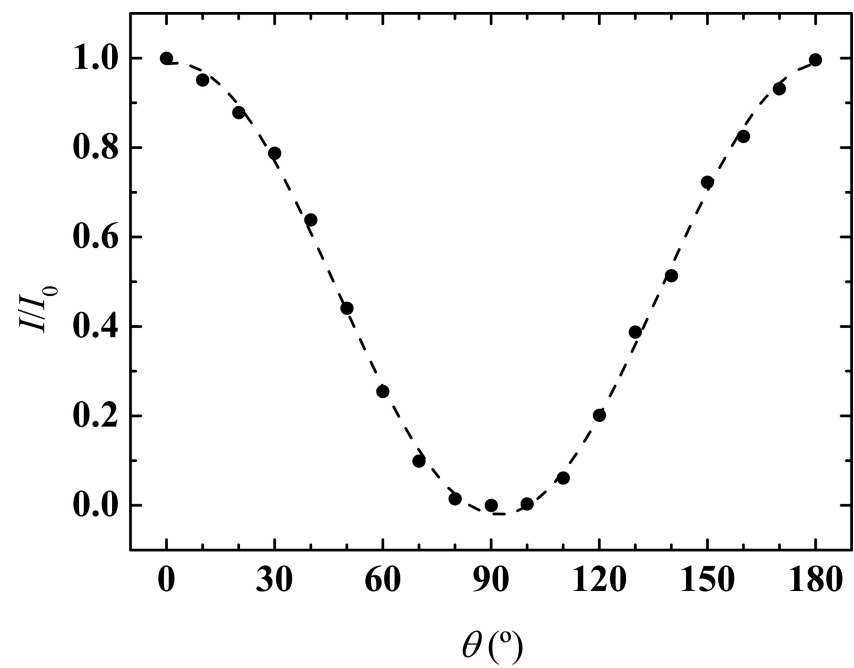

Figura 6: Curva de intensidade relativa em função do ângulo entre os eixos de polarização dos dois polarizadores. Os pontos sólidos circulares são as medidas experimentais e a linha tracejada é o ajuste teórico para a Lei de Malus. onde A', B' e C' são constantes e suas interpretações são muito similares ao discutido no experimento anterior. A constante $\mathrm{A}^{\prime}$ também ajusta o valor mínimo de intensidade e é esperado que seja o mais próximo possível de zero. A constante $\mathrm{B}^{\prime}$ também ajusta o valor máximo da intensidade que, neste caso de intensidade relativa, é esperado que seja próximo a um. A constante $\mathrm{C}^{\prime}$ ajusta a posição angular em que ocorre o máximo de intensidade relativa, esperada próxima a zero. Os valores obtidos são:

$$
\begin{aligned}
& \mathrm{A}^{\prime}=-0,020 \pm 0,008 \\
& \mathrm{~B}^{\prime}=1,01 \pm 0,02 \\
& \mathrm{C}^{\prime}=(-2,2 \pm 0,4)^{\circ}
\end{aligned}
$$

Finalmente, no experimento para verificar a lei fotométrica da distância, a iluminância é medida em função da distância entre a fonte e fotodetector, sendo que a dependência entre elas é dada por:

$$
E=\frac{I}{r^{2}}
$$

onde $E$ é a iluminância, $I$ é a intensidade luminosa da lâmpada e $r$ é a distância entre fonte e detector. A Figura 7 apresenta os resultados obtidos das medidas utilizando-se uma lâmpada incandescente como fonte, assim como a linearização e o ajuste de reta obtidos a partir dos dados experimentais.

É possível observar que os dados obtidos são consistentes. O ajuste dos dados experimentais linearizados foi feito através da seguinte equação:

$$
E=\mathrm{A}^{\prime \prime}+\mathrm{B}^{\prime \prime} \cdot r^{-2}
$$

onde $\mathrm{A}^{\prime \prime}$ e $\mathrm{B}^{\prime \prime}$ são constantes, cujos valores obtidos são:

$$
\begin{aligned}
& \mathrm{A}^{\prime \prime}=(0,174 \pm 0,009) \operatorname{lux} \\
& \mathrm{B}^{\prime \prime}=(0,1235 \pm 0,0004) \operatorname{lm}
\end{aligned}
$$

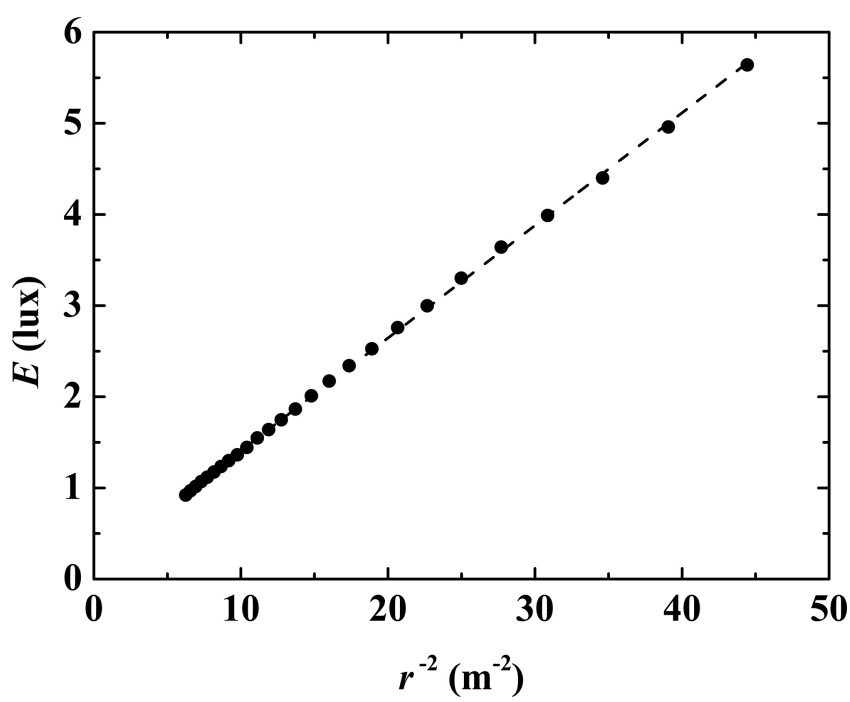

Figura 7: Curva linearizada de iluminância em função da posição. Os pontos sólidos circulares são as medidas experimentais e a linha tracejada é o ajuste linear. 
Do coeficiente angular, pode-se calcular o valor da intensidade luminosa da lâmpada, lembrando que este valor não deve ser o real devido ao luxímetro não estar calibrado.

Finalmente, conclui-se que o luxímetro construído atende as necessidades do laboratório didático de física experimental voltado a experimentos de Óptica. Além disso, o equipamento tem custo relativamente baixo, sua manutenção não necessita mão-de-obra especializada e pode ser excelente exercício de instrumentação científica para a graduação em Física.

\section{Conclusões}

Ao se trabalhar com a experiência "Difração da Luz" no laboratório didático de física experimental, há a necessidade de utilização de instrumentos sensíveis para a medição e análise quantitativa da luz difratada, particularmente luxímetros. Tendo como objetivo a construção de um luxímetro baseado em plataforma aberta Arduino, verificou-se que o mesmo atende aos requisitos necessários para sua aplicação neste experimento trabalhado em sala de aula, possibilitando a substituição de luxímetros comerciais em final de vida útil. Dessa forma, além de custos mais baixos de aquisição e manutenção, sua construção torna-se um excelente exercício de instrumentação a alunos de graduação tanto em Física quanto em Engenharias. Adicionalmente, o equipamento construído foi utilizado em outros experimentos que envolvem luxímetro: "Lei de Malus" e "Lei Fotométrica da Distância". Conclui-se, deste trabalho, que o luxímetro construído utilizando o fotodetector TSL237s-LF e plataforma Arduino atende às necessidades do laboratório didático direcionado a experimentos de Óptica em nível dos cursos de graduação em Física e Engenharias. O equipamento é suficientemente preciso para medir intensidades luminosas até o segundo máximo secundário da curva de difração de luz laser em fenda única, assim como permite a verificação da Lei de Malus em polarizadores e a Lei Fotométrica da Distância utilizando fontes de luz convencionais. Assim, o luxímetro construído pode ser utilizado em sala de aula, em substituição a luxímetros comerciais mais custosos tanto na aquisição quanto na manutenção.

\section{Agradecimentos}

Os autores gostariam de agradecer ao Conselho Nacional de Desenvolvimento Científico e Tecnológico - CNPq, através do Programa Institucional de Bolsas de Iniciação Tecnológica - PIBITI.

\section{Material suplementar}

O seguinte material suplementar está disponível online. Códigos Arduino

\section{REFERÊNCIAS}

[1] H.D. Young e R.A. Freedman, Física IV: Ótica e Física Moderna (Pearson Universidades, São Paulo, 2015).

[2] J.L.P. Ribeiro e M.F.S. Verdeaux, Rev. Bras. Ens. Fis. 34, 1 (2012).

[3] E. Hecht, Optics (Addison-Wesley, New York, 2002).

[4] PASCO, Instruction Sheet for the PASCO Model OS8020, (1994).

[5] M. Moreira, M. Romeu, F.R. Alves e F.R. da Silva, Cad. Bras. Ens. Fís. 35, 721 (2018).

[6] L.S. Pedroso, J.A. Macêdo, M.S.T de Araújo e M.R. Voelzke, Rev. Bras. Ens. Fis. 38, e2503 (2016).

[7] Patrik, Highly Sensitive Arduino Light Sensor, disponível em https://www.instructables.com/id/Highly-sens itive-Arduino-light-sensor/, acessado em 23/11/2020.

[8] AMS, TSL237 High-Sensitivity Light-to-Frequency Converter, disponível em https://br.mouser.com/datasheet/ 2/588/TSL237_DS000156_3-00-1513434.pdf, acessado em $23 / 11 / 2020$.

[9] D.A. Johnson, Light to Frequency converter circuit, disponível em http://www.circuitstoday.com/light-to-f requency-converter-circuit, acessado em 23/11/2020.

[10] D.A. Johnson, Light to Frequency converter circuit, disponível em http://www.discovercircuits.com/H-C orner/lite-freq-con.htm, acessado em 23/11/2020.

[11] M.V. Klein e T.E. Furtak, Optics (John Wiley and Sons, New York, 1986).

[12] JDSU, JDSU Helium-Neon gas laser, disponível em ht tps://www.laser2000.de/en/gas-lasers/248-jdsu-helium - neon-gas-laser.html, acessado em 23/11/2020.

[13] PASCO, Diffraction Plate OS-8850, disponível em https: //www.pasco.com/products/lab-apparatus/light-and-o ptics/diffraction/os-8850, acessado em 23/11/2020. 